\title{
Stabilization and Synchronization of Memristive Chaotic Circuits by Impulsive Control
}

\author{
Limin Zou, ${ }^{1}$ Yang Peng, ${ }^{2}$ Yuming Feng, ${ }^{3}$ and Zhengwen $\mathrm{Tu}^{3}$ \\ ${ }^{1}$ School of Mathematics and Statistics, Chongqing Technology and Business University, Chongqing 400067, China \\ ${ }^{2}$ School of Mathematics and Statistics, Chongqing Three Gorges University, Chongqing 404100, China \\ ${ }^{3}$ Key Laboratory of Intelligent Information Processing and Control, Chongqing Three Gorges University, \\ Chongqing 404100, China \\ Correspondence should be addressed to Limin Zou; limin-zou@163.com
}

Received 14 April 2017; Revised 26 June 2017; Accepted 6 November 2017; Published 5 December 2017

Academic Editor: Sigurdur F. Hafstein

Copyright (C) 2017 Limin Zou et al. This is an open access article distributed under the Creative Commons Attribution License, which permits unrestricted use, distribution, and reproduction in any medium, provided the original work is properly cited.

The purpose of this note is to study impulsive control and synchronization of memristor based chaotic circuits shown by Muthuswamy. We first establish a less conservative sufficient condition for the stability of memristor based chaotic circuits. After that, we discuss the effect of errors on stability. Meanwhile, we also discuss impulsive synchronization of two memristor based chaotic systems. Our results are more general and more applicable than the ones shown by Yang, Li, and Huang. Finally, several numerical examples are given to show the effectiveness of our methods.

\section{Introduction}

The memristor was postulated as the fourth circuit element by Chua $[1,2]$ and was realized by HP Labs $[2,3]$. Memristor has widely potential applications in electronic circuits, computer memory, reconfigurable computing, and so on [4-7]. Recently, implementation of memristor based chaotic circuits is an active topic of research. For example, some memristor based chaotic circuits were proposed by Itoh and Chua [8] and Muthuswamy and Kokate [9]. Memristor based chaotic circuits have applications in many fields; for example, a memristor based chaotic circuit for image encryption was proposed by Lin and Wang [10].

In practical applications, impulsive control has some advantages: for example, impulsive control provides an efficient way in dealing with systems especially which cannot endure continuous control inputs. During the last several decades, impulsive control theory has attracted considerable attention because impulsive control method can be employed in many fields, such as the stabilization and synchronization of chaotic systems [11-14] and complex dynamical networks [15-19]. For more results on impulsive control and its applications, the reader is referred to $[11,20,21]$ and the references therein.

Recently, complex dynamical systems are receiving much attention, and there is no exception for chaotic systems. Muthuswamy [22] provided a practical implementation of a memristor based chaotic circuit. By applying impulsive control theory, Yang et al. [23] obtained some sufficient conditions for the asymptotic stabilization and synchronization of the memristor based chaotic system shown in [22].

In this note, we shall also consider the asymptotic stabilization and synchronization of memristor based chaotic circuits, as in [22]. We first derive a less conservative sufficient condition for the stability of the memristor based chaotic circuits shown by Muthuswamy [22]. In many practical applications, we cannot guarantee the systems without any error due to the limit of equipment and technology. For this reason, we discuss the effect of errors on stability in this note. Meanwhile, we also discuss impulsive synchronization of two memristor based chaotic systems. Compared with the results shown in [23], our methods are more general and more applicable. Finally, we give some numerical examples which show the effectiveness of our methods. 


\section{Memristor Based Chaotic Circuit and Its Equivalent Form}

The equations for the memristor based chaotic circuit presented in [22] are described by

$$
\begin{aligned}
\frac{d v_{1}(t)}{d t} & =\frac{1}{C_{1}}\left(\frac{v_{2}(t)-v_{1}(t)}{R}-i(t)\right), \\
\frac{d v_{2}(t)}{d t} & =\frac{1}{C_{2}}\left(\frac{v_{1}(t)-v_{2}(t)}{R}-i_{L}(t)\right), \\
\frac{d i_{L}(t)}{d t} & =\frac{v_{2}(t)}{L} \\
\frac{d \phi(t)}{d t} & =v_{1}(t)
\end{aligned}
$$

where

$$
i(t)=W(\phi(t)) v_{1}(t)=\frac{d q}{d \phi} v_{1}(t)
$$

The author of [22] chose a cubic nonlinearity for the $q-\phi$ function:

$$
q(\phi)=\alpha \phi+\beta \phi^{3}
$$

and so the memductance function $W(\phi)$ is given by

$$
W(\phi)=\frac{d q}{d \phi}=\alpha+3 \beta \phi^{2}
$$

We choose the system parameters as

$$
\begin{aligned}
\alpha & =-0.663 \cdot 10^{-3}, \\
\beta & =0.004 \cdot 10^{-3}, \\
R & =2 \mathrm{k} \Omega, \\
L & =15.8 \mathrm{H}, \\
C_{1} & =6.1 \mu \mathrm{F}, \\
C_{2} & =71 \mu \mathrm{F},
\end{aligned}
$$

which make system (1) chaotic [22, 23]. Figure 1 shows the chaotic phenomenon of this system with the initial condition $\left(v_{1}(0), v_{2}(0), i_{L}(0), \phi(0)\right)^{T}=(0.1302,0.0924,0.0078,0.1253)^{T}$.

In the sequel, we mainly adopt the notation and terminology in [23]. To analyze the asymptotic stabilization of system (1), we let

$$
\begin{aligned}
& x_{3}=i_{L}, \\
& x_{4}=\phi, \\
& a_{1}=\frac{1}{C_{1}}, \\
& a_{2}=\frac{1}{C_{2}}, \\
& a_{3}=\frac{1}{R}, \\
& a_{4}=\frac{1}{L} .
\end{aligned}
$$

Then the memristor based chaotic circuit (1) can be rewritten as

$$
\begin{aligned}
& \frac{d x_{1}}{d t}=a_{1}\left(a_{3}\left(x_{2}-x_{1}\right)-W\left(x_{4}\right) x_{1}\right), \\
& \frac{d x_{2}}{d t}=a_{2}\left(a_{3}\left(x_{1}-x_{2}\right)-x_{3}\right), \\
& \frac{d x_{3}}{d t}=a_{4} x_{2}, \\
& \frac{d x_{4}}{d t}=x_{1},
\end{aligned}
$$

which is equivalent to

$$
\begin{aligned}
& \frac{d x_{1}}{d t}=a_{1}\left(a_{3}\left(x_{2}-x_{1}\right)-\alpha x_{1}-3 \beta x_{1} x_{4}^{2}\right) \\
& \frac{d x_{2}}{d t}=a_{2}\left(a_{3}\left(x_{1}-x_{2}\right)-x_{3}\right) \\
& \frac{d x_{3}}{d t}=a_{4} x_{2} \\
& \frac{d x_{4}}{d t}=x_{1} .
\end{aligned}
$$

By decomposing the linear and nonlinear parts of the memristor based chaotic circuit system in (8), we can rewrite it as

$$
\dot{X}=A X+\psi(X) \text {, }
$$

where

$$
\begin{aligned}
X & =\left[x_{1}, x_{2}, x_{3}, x_{4}\right]^{T}, \\
\psi(X) & =\left[-3 a_{1} \beta x_{1} x_{4}^{2}, 0,0,0\right]^{T}, \\
A & =\left[\begin{array}{cccc}
-a_{1}\left(\alpha+a_{3}\right) & a_{1} a_{3} & 0 & 0 \\
a_{2} a_{3} & -a_{2} a_{3} & -a_{2} & 0 \\
0 & a_{4} & 0 & 0 \\
1 & 0 & 0 & 0
\end{array}\right] .
\end{aligned}
$$



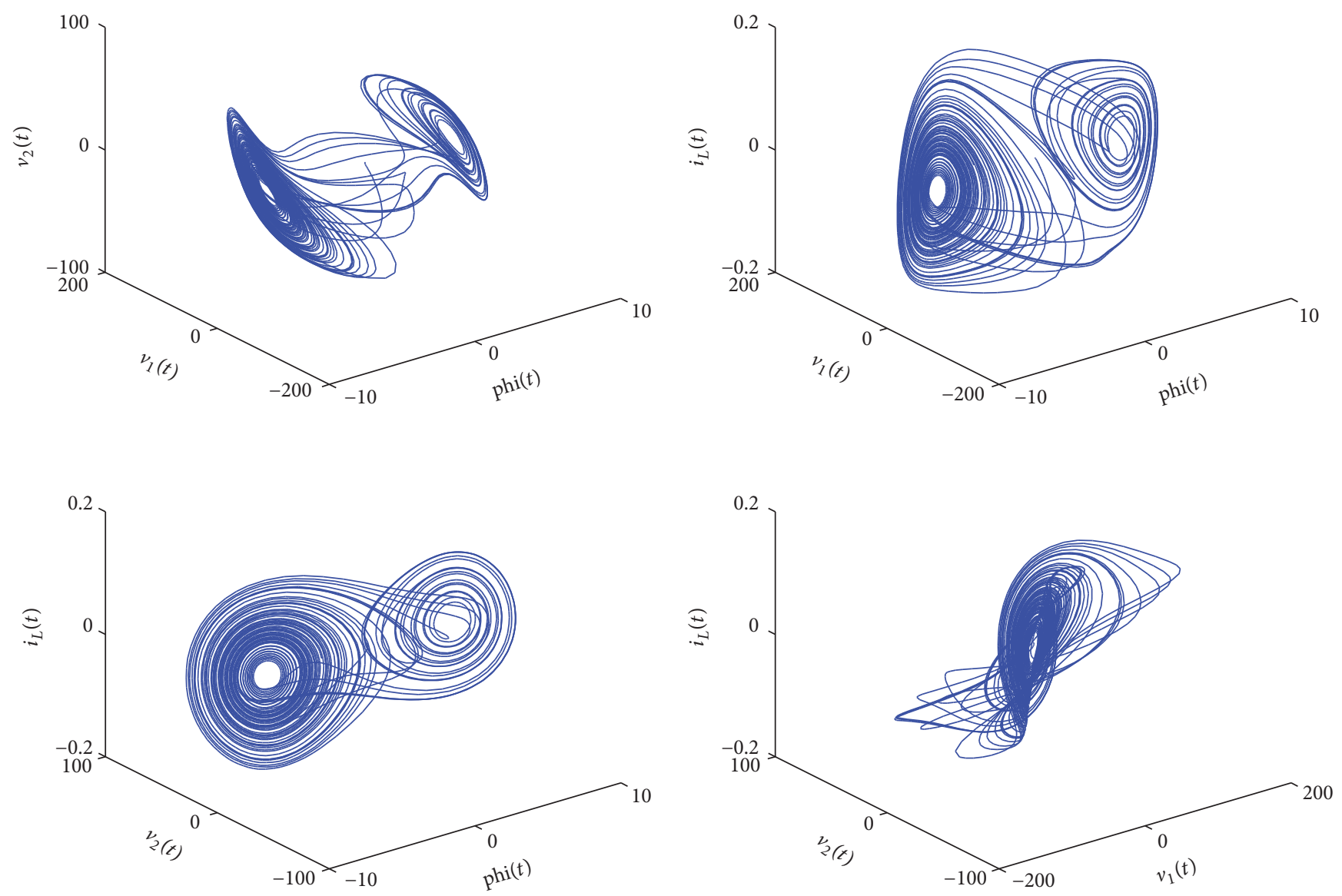

FIGURE 1: The state trajectory of the memristor based chaotic circuit shown in (1).

The impulsively controlled memristor based chaotic circuit is given by

$$
\begin{aligned}
\dot{X} & =A X+\psi(X), \quad t \neq \tau_{k}, \\
\Delta X & =B X, \quad t=\tau_{k}, \quad k=1,2, \ldots,
\end{aligned}
$$

where $\tau_{k}$ denote the moments when impulsive control occurs and $B \in R^{n \times n}$ is impulsive control gain. Without loss of generality, we assume that $t_{0}<\tau_{1}<\tau_{2}<\cdots, \lim _{k \rightarrow \infty} \tau_{k}=$ $\infty$.

\section{Impulsive Control of the Memristor Based Chaotic Circuit Shown by Muthuswamy}

In this section, we design impulsive control for the memristor based chaotic circuit shown by Muthuswamy.

Theorem 1. Let $\lambda_{1}$ be the largest eigenvalue of $(I+B)^{T}(I+B)$ and suppose that $\lambda_{2}$ is the largest eigenvalues of $A+A^{T}$. Then the origin of impulsive control system (11) is asymptotically stable if

$$
\lambda_{2}\left(\tau_{k+1}-\tau_{k}\right) \leq-\ln \left(\gamma \lambda_{1}\right), \quad \gamma>1 .
$$

Proof. Let us construct the following Lyapunov function:

$$
V(t, X)=X^{T} X
$$

It is easy to verify that conditions 1 and 4 of Theorem 3.1.3 in [11] are satisfied. When $t \neq \tau_{k}$, we have

$$
\begin{aligned}
D^{+} V(t, X) & =X^{T}\left(A+A^{T}\right) X+2 X^{T} \psi(X) \\
& =X^{T}\left(A+A^{T}\right) X-6 a_{1} \beta x_{1}^{2} x_{4}^{2} \\
& \leq X^{T}\left(A+A^{T}\right) X \leq \lambda_{2} X^{T} X .
\end{aligned}
$$

Hence, condition 2 of Theorem 3.1.3 in [11] is satisfied with

$$
g(t, \omega)=\left(\lambda_{2}-2 a_{1} \alpha\right) \omega
$$

From the fact that

$$
\|X+U(k, X)\|=\|X+B X\| \leq\|I+B\|\|X\|
$$


and $\|I+B\|$ is finite, we know that there exists a $\rho_{0}>0$ such that $X \in S_{\rho_{0}}$, which implies that $X+U(k, X) \in S_{\rho}$ for all $k$. When $t=\tau_{k}$, we have

$$
\begin{aligned}
D^{+} V\left(\tau_{k}, X+B X\right) & =(X+B X)^{T}(X+B X) \\
& =X^{T}(I+B)^{T}(I+B) X \\
& \leq \lambda_{1} X^{T} X .
\end{aligned}
$$

Hence, condition 3 of Theorem 3.1.3 in [11] is satisfied with

$$
\chi_{k}(\omega)=\lambda_{1} \omega
$$

It follows from Theorem 3.1.3 in [11] that the asymptotic stability of the impulsive control system (11) is implied by that of the following comparison system:

$$
\begin{aligned}
\dot{\omega}(t) & =\left(\lambda_{2}-2 a_{1} \alpha\right) \omega, \quad t \neq \tau_{k}, \\
\omega\left(\tau_{k}^{+}\right) & =\lambda_{1} \omega\left(\tau_{k}\right), \\
\omega\left(\tau_{0}^{+}\right) & =\omega_{0} \geq 0 .
\end{aligned}
$$

It follows from Theorem 3.1.4 in [11] that if

$$
\left(\lambda_{2}-2 a_{1} \alpha\right)\left(\tau_{k+1}-\tau_{k}\right) \leq-\ln \left(\gamma \lambda_{1}\right), \quad \gamma>1
$$

is satisfied, then the origin of (11) is asymptotically stable. This completes the proof.

Remark 2. Let

$$
\widetilde{A}=\left[\begin{array}{cccc}
-a_{1} a_{3} & a_{1} a_{3} & 0 & 0 \\
a_{2} a_{3} & -a_{2} a_{3} & -a_{2} & 0 \\
0 & a_{4} & 0 & 0 \\
1 & 0 & 0 & 0
\end{array}\right]
$$

and suppose that $\widetilde{\lambda}_{2}$ is the largest eigenvalues of $\widetilde{A}+\widetilde{A}^{T}$. Yang et al. showed in [23] that the origin of impulsive control system (11) is asymptotically stable if

$$
\begin{array}{r}
\left(\tilde{\lambda}_{2}+2 \sqrt{\sup \left\{a_{1}^{2} W^{2}\left(x_{4}\right)\right\}}\right)\left(\tau_{k+1}-\tau_{k}\right) \leq-\ln \left(\gamma \lambda_{1}\right) \\
\gamma>1 .
\end{array}
$$

Since

$$
\begin{aligned}
\lambda_{2} & =\lambda_{\max }\left(A+A^{T}\right) \\
& =\lambda_{\max }\left(\widetilde{A}+\widetilde{A}^{T}+2 \operatorname{diag}\left(-a_{1} \alpha, 0,0,0\right)\right) \\
& \leq \lambda_{\max }\left(\widetilde{A}+\widetilde{A}^{T}\right)+\lambda_{\max }\left(2 \operatorname{diag}\left(-a_{1} \alpha, 0,0,0\right)\right) \\
& =\widetilde{\lambda}_{2}-2 a_{1} \alpha \leq \widetilde{\lambda}_{2}+2 \sqrt{\sup \left\{a_{1}^{2} W^{2}\left(x_{4}\right)\right\}},
\end{aligned}
$$

our result is less conservative than Theorem 1 of [23]. Meanwhile, our method is also simpler than Theorem 1 of [23], because we do not need to calculate the supremum of $\left|W\left(x_{4}\right)\right|$.

In many practical applications, we cannot guarantee the impulses without any error due to the limit of equipment and technology. So we should take into account the influence of impulsive control gain errors on the systems. Motivated by the above discussions, we will study the stabilization of system (11) with bounded impulsive control gain error. The corresponding system can be described as

$$
\begin{aligned}
\dot{X} & =A X+\psi(X), \quad t \neq \tau_{k}, \\
\Delta X & =(B+\Delta B) X, \quad t=\tau_{k}, \quad k=1,2, \ldots,
\end{aligned}
$$

where $\Delta B$ is gain error, which is often time-varying and bounded. As pointed out in $[21,24]$, we can assume that $\Delta B=m F(t) B, m \geq 0$, and $F^{T}(t) F(t) \leq I$.

Theorem 3. Let $\lambda_{1}$ be the largest eigenvalue of

$$
(1+\varepsilon)\left(I+B+B^{T}\right)+\left(1+\varepsilon+m^{2}\left(1+\frac{1}{\varepsilon}\right)\right) B^{T} B
$$

where $\varepsilon>0$ and suppose that $\lambda_{2}$ is the largest eigenvalues of $A+A^{T}$. Then the origin of impulsive control system (24) is asymptotically stable if

$$
\lambda_{2}\left(\tau_{k+1}-\tau_{k}\right) \leq-\ln \left(\gamma \lambda_{1}\right), \quad \gamma>1 .
$$

Proof. Let us construct the following Lyapunov function:

$$
V(t, X)=X^{T} X
$$

When $t=\tau_{k}$, we have

$$
\begin{aligned}
& D^{+} V\left(\tau_{k}, X+B X\right)=X^{T}(C+\Delta B)^{T}(C+\Delta B) X \\
& \quad \leq X^{T}\left((1+\varepsilon) C^{T} C+\left(1+\frac{1}{\varepsilon}\right)(\Delta B)^{T} \Delta B\right) X \\
& \quad \leq X^{T}\left((1+\varepsilon) C^{T} C+m^{2}\left(1+\frac{1}{\varepsilon}\right) B^{T} B\right) X \\
& \quad \leq \lambda_{1} X^{T} X^{T},
\end{aligned}
$$

where $C=I+B$. The rest of proof is the same as that of Theorem 1, so we omit it here for simplicity. This completes the proof.

In many practical applications, the parameters $a_{1}=$ $1 / C_{1}, a_{2}=1 / C_{2}, a_{3}=1 / R$, and $a_{4}=1 / L$ may also contain errors. In what follows, we will consider system (24) with parameter uncertainty. The corresponding system can be described as

$$
\begin{aligned}
\dot{X} & =(A+\Delta A) X+\psi(X), \quad t \neq \tau_{k}, \\
\Delta X & =(B+\Delta B) X, \quad t=\tau_{k}, \quad k=1,2, \ldots,
\end{aligned}
$$


where $\triangle A$ is the parametric uncertainty and has the following form: $\Delta A=\mu G(t) A, G(t) G^{T}(t) \leq I, \mu \geq 0$.

Theorem 4. Let $\lambda_{1}$ be the largest eigenvalue of

$$
(1+\varepsilon)\left(I+B+B^{T}\right)+\left(1+\varepsilon+m^{2}\left(1+\frac{1}{\varepsilon}\right)\right) B^{T} B,
$$

where $\varepsilon>0$ and suppose that $\lambda_{2}$ is the largest eigenvalues of $\left(A+A^{T}\right)+\mu\left(A^{T} A+I\right)$. Then the origin of impulsive control system (29) is asymptotically stable if

$$
\lambda_{2}\left(\tau_{k+1}-\tau_{k}\right) \leq-\ln \left(\gamma \lambda_{1}\right), \quad \gamma>1 .
$$

Proof. Let us construct the following Lyapunov function:

$$
V(t, X)=X^{T} X
$$

When $t \neq \tau_{k}$, we have

$$
\begin{aligned}
D^{+} V(t, X) & =X^{T}\left(C+C^{T}\right) X-6 a_{1} \beta x_{1}^{2} x_{4}^{2} \\
& \leq X^{T}\left(C+C^{T}\right) X \\
& \leq X^{T}\left(\left(A+A^{T}\right)+m\left(A^{T} A+I\right)\right) X \\
& \leq \lambda_{2} X^{T} X,
\end{aligned}
$$

where $C=A+\Delta A$. The rest of proof is the same as that of Theorem 1, so we omit it here for simplicity. This completes the proof.

\section{Impulsive Synchronization of the Memristor Based Chaotic Circuit Shown by Muthuswamy}

In this section, we investigate impulsive synchronization of two memristor based chaotic circuits. Equation (9) is the driving system and the driven system is defined as

$$
\begin{aligned}
\dot{Y} & =A Y+\psi(Y), \quad t \neq \tau_{k}, \\
\Delta Y & =-B e, \quad t=\tau_{k}, \quad k=1,2, \ldots,
\end{aligned}
$$

where $B \in R^{n \times n}$ is impulsive control gain and $e^{T}=\left(e_{1}, e_{2}\right.$, $\left.e_{3}, e_{4}\right)=\left(x_{1}-y_{1}, x_{2}-y_{2}, x_{3}-y_{3}, x_{4}-y_{4}\right)$ is the synchronization error. Then the error system of the impulsive synchronization is given by

$$
\begin{aligned}
\dot{e} & =A e+\phi(X)-\phi(Y), \quad t \neq \tau_{k}, \\
\Delta e & =B e, \quad t=\tau_{k}, \quad k=1,2, \ldots
\end{aligned}
$$

Note that

$$
\begin{aligned}
\phi(X)-\phi(Y) & =-3 a_{1} \beta\left[x_{1} x_{4}^{2}-y_{1} y_{4}^{2}, 0,0,0\right]^{T} \\
& =-3 a_{1} \beta N(X, Y) e
\end{aligned}
$$

where

$$
N(X, Y)=\left[\begin{array}{cccc}
x_{4}^{2} & 0 & 0 & y_{1}\left(x_{4}+y_{4}\right) \\
0 & 0 & 0 & 0 \\
0 & 0 & 0 & 0 \\
0 & 0 & 0 & 0
\end{array}\right] .
$$

The eigenvalues of $N(X, Y)+N^{T}(X, Y)$ are

$$
\begin{aligned}
& \mu_{1}=\mu_{2}=0, \\
& \mu_{3}=x_{4}^{2}-\sqrt{x_{4}^{4}+y_{1}^{2}\left(x_{4}+y_{4}\right)^{2}} \leq 0, \\
& \mu_{4}=x_{4}^{2}+\sqrt{x_{4}^{4}+y_{1}^{2}\left(x_{4}+y_{4}\right)^{2}} \geq 0 .
\end{aligned}
$$

From Figure 1, we know that the state variable of (11) is bounded and suppose that $\left|x_{1}\right| \leq M_{1},\left|x_{4}\right| \leq M_{4}$.

Theorem 5. Let $\lambda_{1}$ be the largest eigenvalue of $(I+B)^{T}(I+$ $B)$ and suppose that $\lambda_{2}$ is the largest eigenvalues of $A+A^{T}$. Then the origin of impulsive synchronization error system (35) is asymptotically stable if

$$
\left(\lambda_{2}+6 a_{1} \beta M_{1} M_{4}\right)\left(\tau_{k+1}-\tau_{k}\right) \leq-\ln \left(\gamma \lambda_{1}\right), \quad \gamma>1 .
$$

Proof. Let us construct the following Lyapunov function:

$$
V(t, e)=e^{T} e .
$$

When $t \neq \tau_{k}$, we have

$$
\begin{aligned}
& D^{+} V(t, e) \\
&=e^{T}\left(A+A^{T}\right) e \\
& \quad-3 a_{1} \beta e^{T}\left(N(X, Y)+N^{T}(X, Y)\right) e \\
& \leq \lambda_{2} e^{T} e-3 a_{1} \beta e^{T}\left(N(X, Y)+N^{T}(X, Y)\right) e \\
& \leq \lambda_{2} e^{T} e-3 a_{1} \beta\left(x_{4}^{2}-\sqrt{x_{4}^{4}+y_{1}^{2}\left(x_{4}+y_{4}\right)^{2}}\right) e^{T} e \\
&=\left(\lambda_{2}+3 a_{1} \beta\left(\sqrt{x_{4}^{4}+y_{1}^{2}\left(x_{4}+y_{4}\right)^{2}}-x_{4}^{2}\right)\right) e^{T} e \\
& \leq\left(\lambda_{2}+3 a_{1} \beta\left|y_{1}\right|\left|x_{4}+y_{4}\right|\right) e^{T} e \\
& \leq\left(\lambda_{2}+6 a_{1} \beta M_{1} M_{4}\right) e^{T} e .
\end{aligned}
$$

The rest of proof is the same as that of Theorem 1, which is omitted here for simplicity. This completes the proof.

\section{Numerical Examples}

In this section, some numerical examples are given to illustrate the effectiveness of our results. The initial condition of the system (11) is $X(0)=(0.1302,0.0924,0.0078,0.1253)^{T}$. 

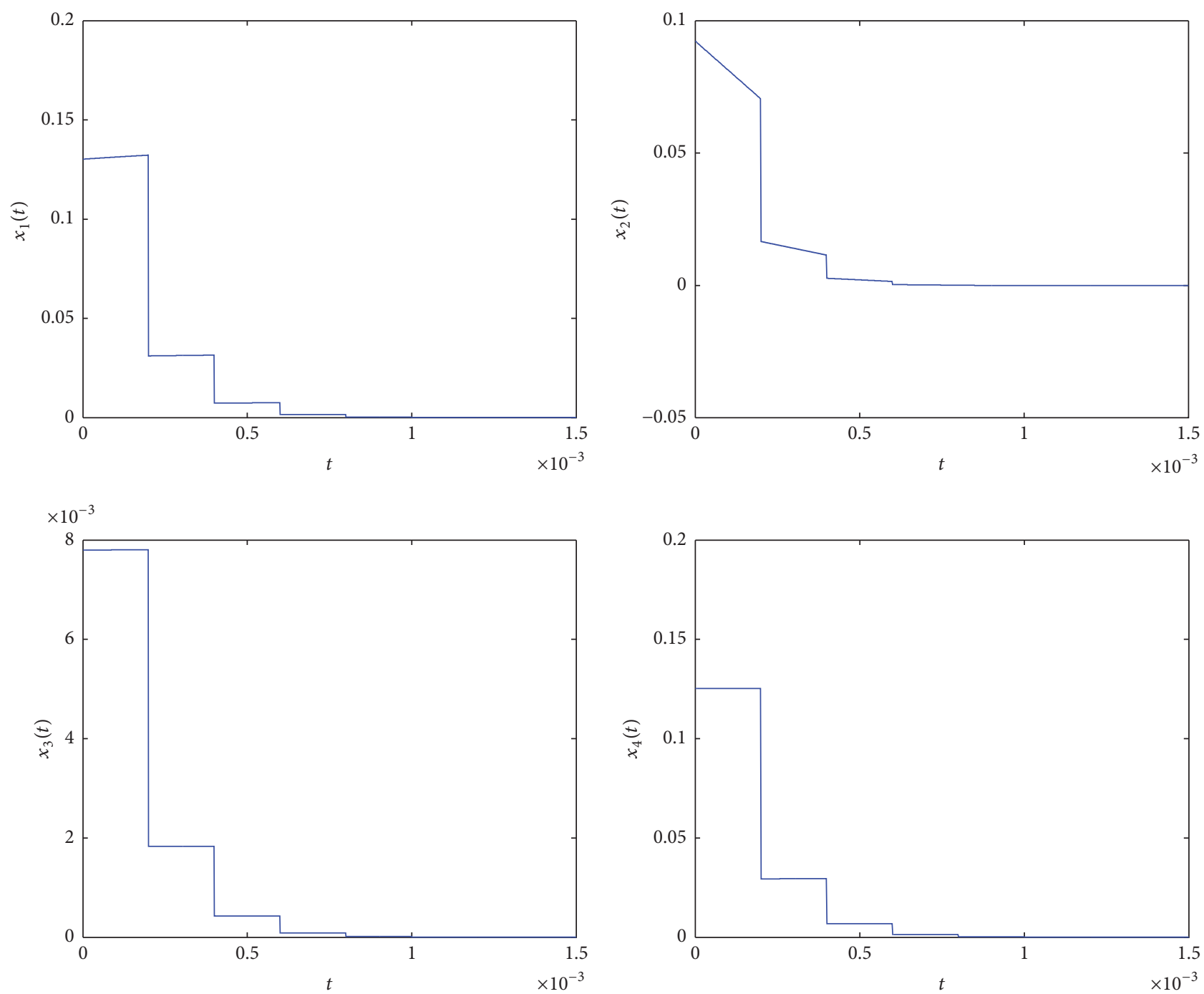

FIGURE 2: The state trajectory of the controlled memristor based chaotic circuit.

Example 1. It is easy to see that

$$
A=\left[\begin{array}{cccc}
26.7213 & 81.9672 & 0 & 0 \\
7.0423 & -7.0423 & -14084.5070 & 0 \\
0 & 0.0633 & 0 & 0 \\
1 & 0 & 0 & 0
\end{array}\right]
$$

and $\lambda_{2}=14077.6856$. In this example, we choose the impulsive control gain matrix $B$ as

$$
B=\left[\begin{array}{cccc}
-0.8000 & 0 & 0 & 0 \\
0 & -0.8000 & 0 & 0 \\
0 & 0 & -0.8000 & 0 \\
0 & 0 & 0 & -0.8000
\end{array}\right]
$$

Then, we have $\lambda_{1}=0.0400$. By Theorem 1 , we know that if

$$
\tau_{k+1}-\tau_{k} \leq-\frac{\ln (0.0400 \gamma)}{14077.6856}, \quad \gamma>1,
$$

holds, then origin of impulsive control system (11) is asymptotically stable. To do this, we choose $\gamma=1.0001$; then we have

$$
\tau_{k+1}-\tau_{k} \leq 0.00022
$$

The simulation results with $\tau_{k+1}-\tau_{k}=0.0002$ are shown in Figure 2.

Example 2. In this example, the coefficient matrix $A$ and the impulsive control gain matrix $B$ are the same as Example 1 . 

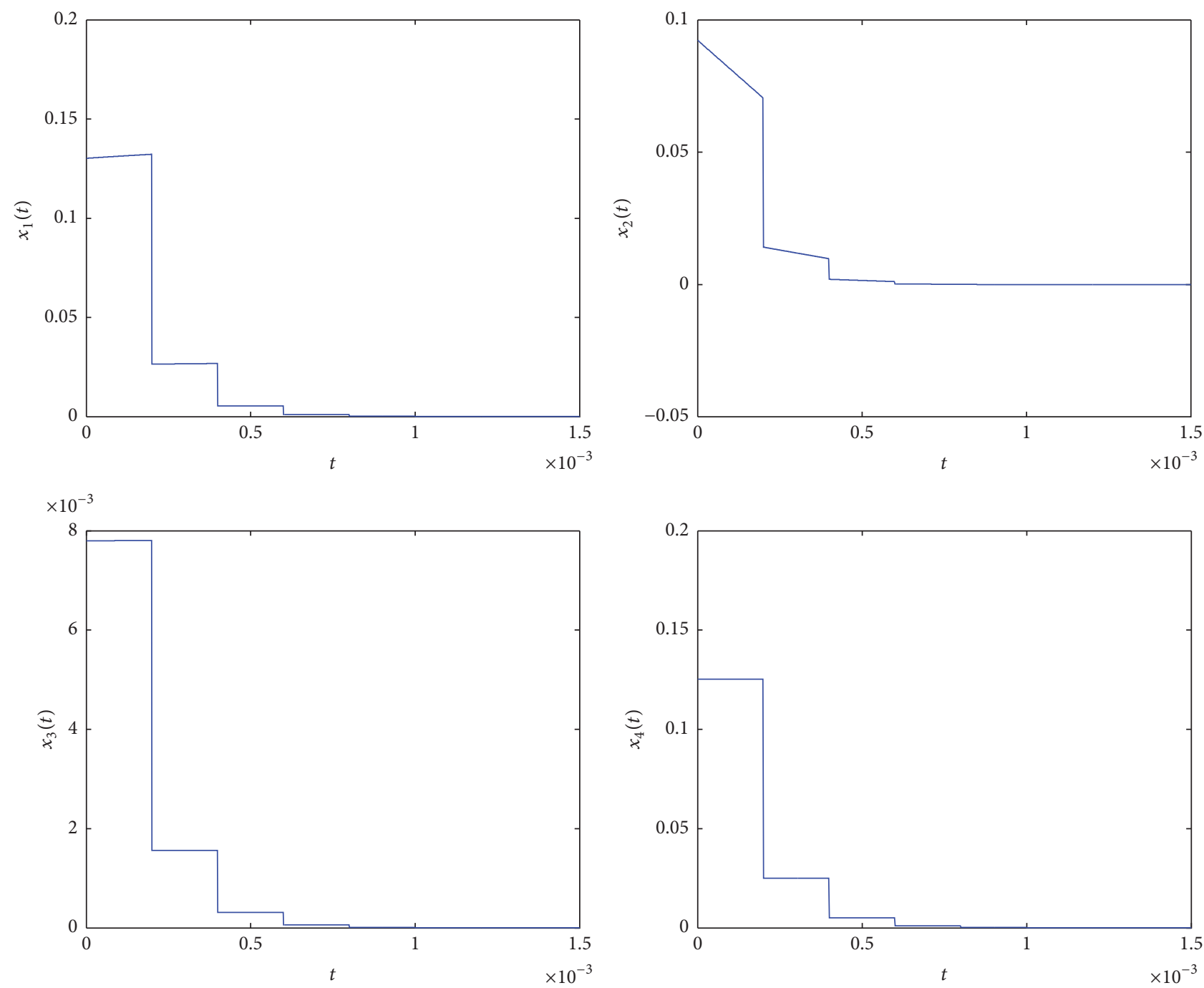

FIGURE 3: The state trajectory of the controlled memristor based chaotic circuit.

Suppose that $\Delta B=0.05 \sin t B$. Then, we have $m=0.05$. By Theorem 3, we know that if

$$
\tau_{k+1}-\tau_{k} \leq-\frac{\ln \left(\gamma \lambda_{1}\right)}{14077.6856}, \quad \gamma>1,
$$

holds, then origin of impulsive control system (24) is asymptotically stable. To do this, we choose $\gamma=1.0001, \varepsilon=0.2100$; then we have $\lambda_{1}=0.0576$ and so

$$
\tau_{k+1}-\tau_{k} \leq 0.00020 .
$$

The simulation results with $\tau_{k+1}-\tau_{k}=0.00020$ are shown in Figure 3.
Example 3. In this example, the matrices $A, B$, and $\Delta B$ are the same as Example 2. For the sake of simplicity, $\Delta A$ is specified as

$$
\Delta A=5 \times 10^{-6} \cos t A .
$$

Then, we have $\mu=0.05$. By Theorem 4 , we know that if

$$
\tau_{k+1}-\tau_{k} \leq-\frac{\ln \left(\gamma \lambda_{1}\right)}{\lambda_{2}}, \quad \gamma>1,
$$

holds, then origin of impulsive control system (29) is asymptotically stable. To do this, we choose $\gamma=1.0001, \varepsilon=0.2100$; then we have $\lambda_{1}=0.0576, \lambda_{2}=14582.0997$ and so

$$
\tau_{k+1}-\tau_{k} \leq 0.00019 .
$$



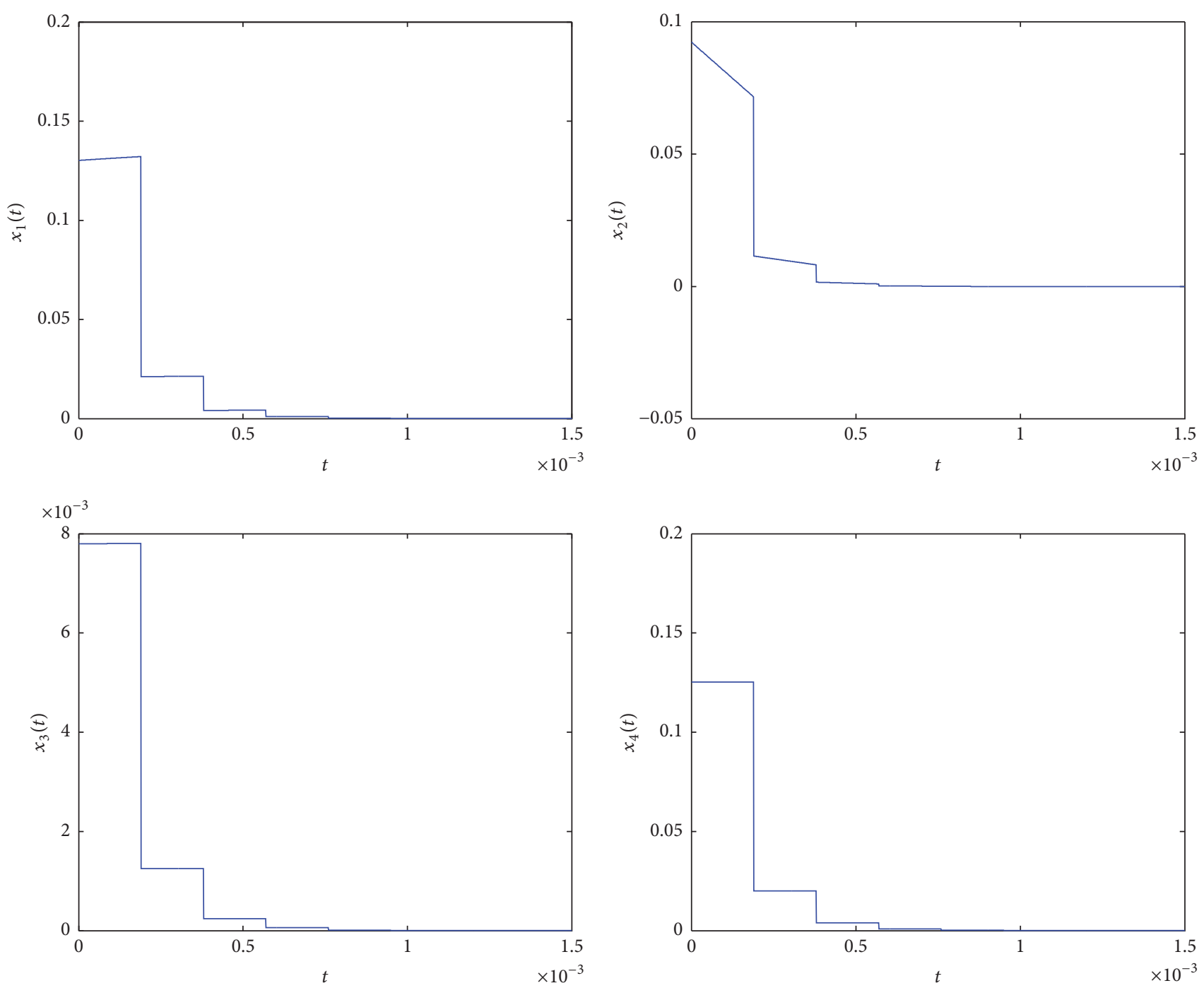

FIGURE 4: The state trajectory of the controlled memristor based chaotic circuit.

The simulation results with $\tau_{k+1}-\tau_{k}=0.00019$ are shown in Figure 4.

Example 4. In this example, the matrix $A$ is the same as Example 1. We choose the matrix $B$ as

$$
B=\left[\begin{array}{cccc}
-1.5000 & 0 & 0 & 0 \\
0 & -1.5000 & 0 & 0 \\
0 & 0 & -1.5000 & 0 \\
0 & 0 & 0 & -1.5000
\end{array}\right]
$$

Then, we have $\lambda_{1}=0.250, \lambda_{2}=14077.6856$. Meanwhile, we know that $\left|x_{1}\right| \leq 200,\left|x_{4}\right| \leq 10$. By Theorem 5, we know that if

$$
\tau_{k+1}-\tau_{k} \leq \frac{-\ln \left(\gamma \lambda_{1}\right)}{\lambda_{2}+6 a_{1} \beta M_{1} M_{4}}, \quad \gamma>1
$$

holds, then the origin of impulsive synchronization error system (35) is asymptotically stable. To do this, we choose $\gamma=1.0001$ and so

$$
\tau_{k+1}-\tau_{k} \leq 0.00006
$$

The initial condition of the driving system (9) is also $X(0)=$ $(0.1302,0.0924,0.0078,0.1253)^{T}$ and the initial condition of the driven system $(35)$ is $Y(0)=(0.3922,0.6787,0.7577$, $0.7431)^{T}$. The simulation results with $\tau_{k+1}-\tau_{k}=0.00006$ are shown in Figure 5.

\section{Conclusion}

In this note, we discuss impulsive control and synchronization of memristor based chaotic circuits shown by Muthuswamy [22]. Our first result is less conservative than Theorem 1 of [23]. Meanwhile, we also discuss the effect of errors on stability, so our results are more general and more applicable than the ones shown in [23]. 

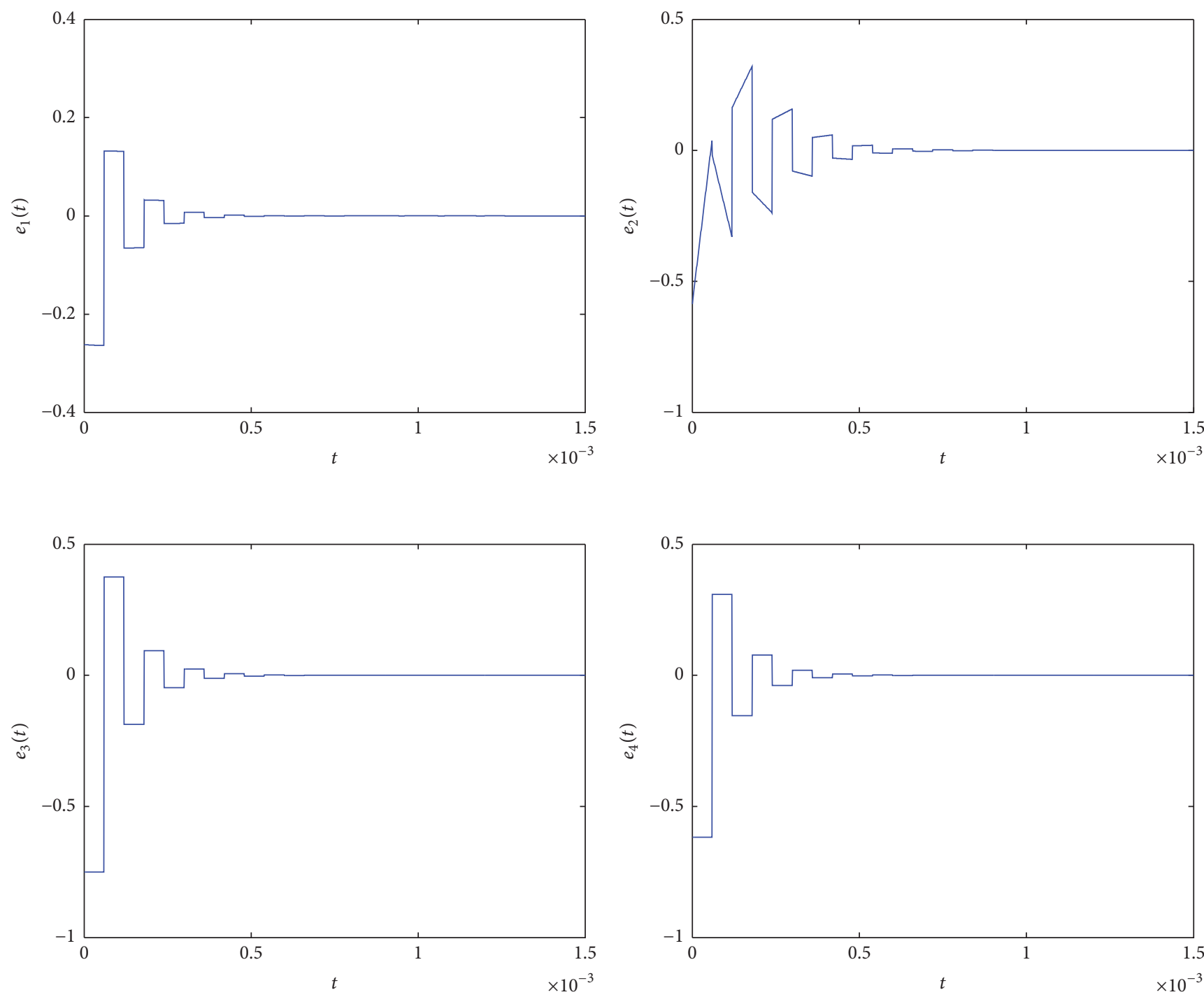

FIGURE 5: Simulation results of synchronization errors.

\section{Conflicts of Interest}

The authors declare that they have no conflicts of interest.

\section{Authors' Contributions}

All authors contributed equally to the writing of this paper. All authors read and approved the final version of this paper.

\section{Acknowledgments}

This work is funded by Chongqing Research Program of Basic Research and Frontier Technology (no. cstc2017jcyjAX0032), the National Natural Science Foundation of China under Grant no 11601047, Key Laboratory of Chongqing Municipal Institutions of Higher Education (Grant no. [2017]3), and project supported by Program of Chongqing Development and Reform Commission (Grant no. 2017[1007]).

\section{References}

[1] L. O. Chua, "Memristor-the missing circuit element," IEEE Transactions on Circuit Theory, vol. 18, no. 5, pp. 507-519, 1971

[2] "Memristor-Wikipedia," https://en.wikipedia.org/wiki/Memristor.

[3] D. B. Strukov, G. S. Snider, D. R. Stewart, and R. S. Williams, "The missing memristor found," Nature, vol. 453, pp. 80-83, 2008.

[4] Y. V. Pershin, S. La Fontaine, and M. Di Ventra, "Erratum: Memristive model of amoeba learning," Physical Review E, vol. 82, no. 1, 2010.

[5] A. Afifi, A. Ayatollahi, and F. Raissi, "Implementation of biologically plausible spiking neural network models on the memristor crossbar-based CMOS/nano circuits," in Proceedings of the ECCTD 2009 - European Conference on Circuit Theory and Design Conference Program, pp. 563-566, Turkey, August 2009.

[6] J. Borghetti, G. S. Snider, P. J. Kuekes, J. J. Yang, D. R. Stewart, and R. S. Williams, “Memristive' switches enable "stateful” logic 
operations via material implication," Nature, vol. 464, no. 7290, pp. 873-876, 2010.

[7] S. H. Jo, T. Chang, I. Ebong, B. B. Bhadviya, P. Mazumder, and W. Lu, "Nanoscale memristor device as synapse in neuromorphic systems," Nano Letters, vol. 10, no. 4, pp. 1297-1301, 2010.

[8] M. Itoh and L. O. Chua, "Memristor oscillators," International Journal of Bifurcation and Chaos, vol. 18, no. 11, pp. 3183-3206, 2008.

[9] B. Muthuswamy and P. Kokate, "Memristor-based chaotic circuits," IETE Technical Review, vol. 26, no. 6, pp. 415-426, 2009.

[10] Z. Lin and H. Wang, "Image encryption based on chaos with PWL memristor in Chua's circuit," in Proceedings of the 2009 International Conference on Communications, Circuits and Systems (ICCCAS), pp. 964-968, Milpitas, Ca, USA, July 2009.

[11] T. Yang, Impulsive Control Theory, Springer, Berlin, Germany, 2001.

[12] T. Yang, L.-B. Yang, and C.-M. Yang, "Impulsive control of Lorenz system," Physica D: Nonlinear Phenomena, vol. 110, no. 1-2, pp. 18-24, 1997.

[13] J. Sun, Y. Zhang, and Q. Wu, "Impulsive control for the stabilization and synchronization of Lorenz systems," Physics Letters A, vol. 298, no. 2-3, pp. 153-160, 2002.

[14] Z. Ai and C. Chen, "Asymptotic stability analysis and design of nonlinear impulsive control systems," Nonlinear Analysis: Hybrid Systems, vol. 24, pp. 244-252, 2017.

[15] C. Li, X. Yu, Z.-W. Liu, and T. Huang, "Asynchronous impulsive containment control in switched multi-agent systems," Information Sciences, vol. 370, pp. 667-679, 2016.

[16] X. Yang, J. Cao, and J. Qiu, "Pth moment exponential stochastic synchronization of coupled memristor-based neural networks with mixed delays via delayed impulsive control," Neural Networks, vol. 65, pp. 80-91, 2015.

[17] X. Yang and J. Lu, "Finite-time synchronization of coupled networks with Markovian topology and impulsive effects," Institute of Electrical and Electronics Engineers Transactions on Automatic Control, vol. 61, no. 8, pp. 2256-2261, 2016.

[18] Q. Song, H. Yan, Z. Zhao, and Y. Liu, "Global exponential stability of complex-valued neural networks with both time-varying delays and impulsive effects," Neural Networks, vol. 79, pp. 108116, 2016.

[19] Q. Song, H. Yan, Z. Zhao, and Y. Liu, "Global exponential stability of impulsive complex-valued neural networks with both asynchronous time-varying and continuously distributed delays," Neural Networks, vol. 81, pp. 1-10, 2016.

[20] W. M. Haddad, V. Chellaboina, and S. G. Nersesov, Impulsive and Hybrid Dynamical Systems: Stability, Dissipativity, and Control, Princeton Series in Applied Mathematics, Princeton University Press, Princeton, NJ, USA, 2006.

[21] T. Ma, Impulsive Control and Synchronization, Science Press, Beijing, China, 2016.

[22] B. Muthuswamy, "Implementing memristor based chaotic circuits," International Journal of Bifurcation and Chaos, vol. 20, no. 5, pp. 1335-1350, 2010.

[23] S. J. Yang, C. D. Li, and T. W. Huang, "Impulsive control and synchronization of memristor-based chaotic circuits," International Journal of Bifurcation and Chaos, vol. 24, no. 12, Article ID 1450162, 12 pages, 2014.

[24] T. D. Ma and F. Y. Zhao, "Impulsive stabilization of a class of nonlinear system with bounded gain error," Chinese Physics B, vol. 23, no. 12, Article ID 120504, 2014. 


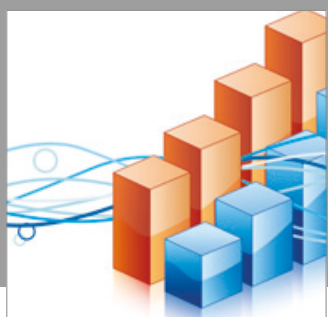

Advances in

Operations Research

vatersals

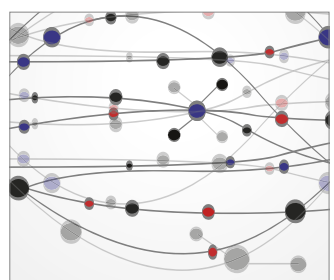

\section{The Scientific} World Journal
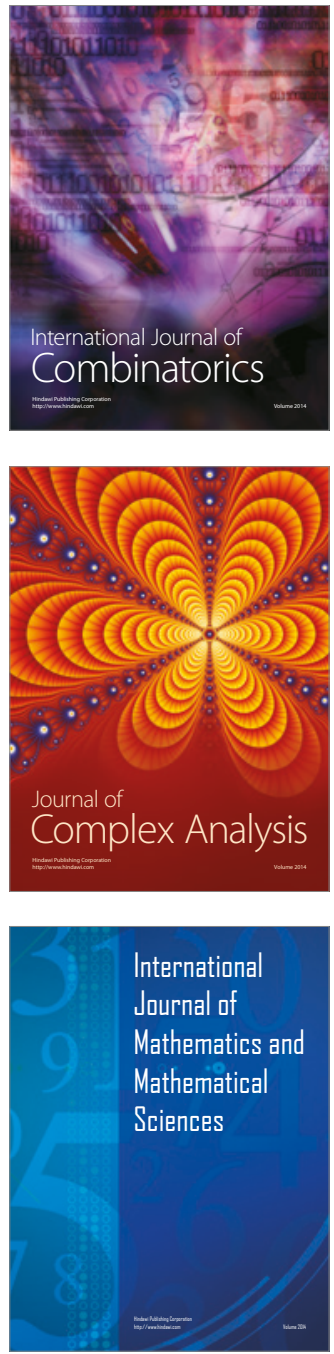
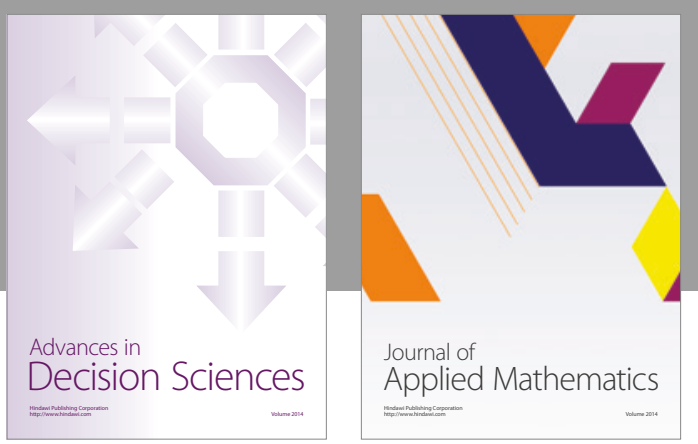

Algebra

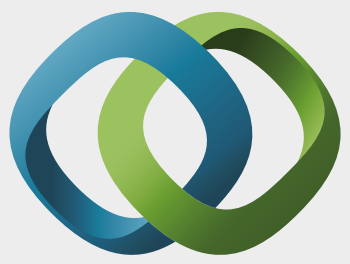

\section{Hindawi}

Submit your manuscripts at

https://www.hindawi.com
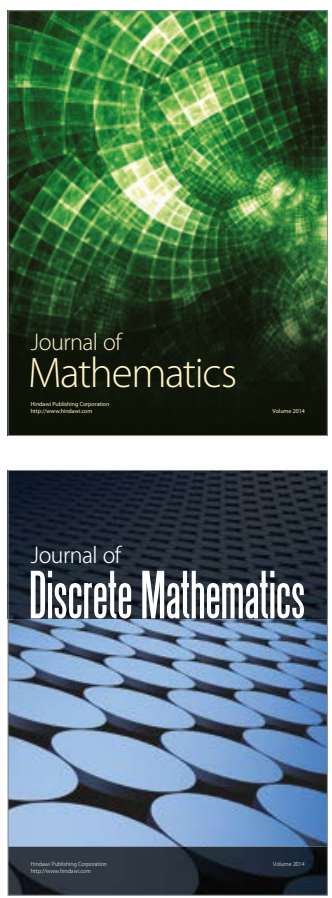

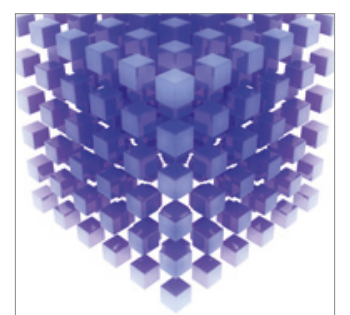

Mathematical Problems in Engineering
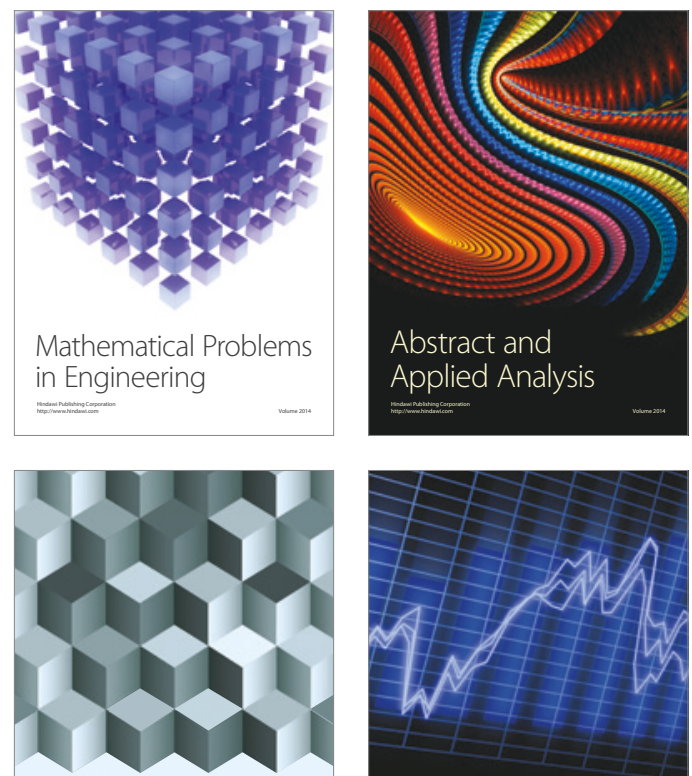

Journal of

Function Spaces

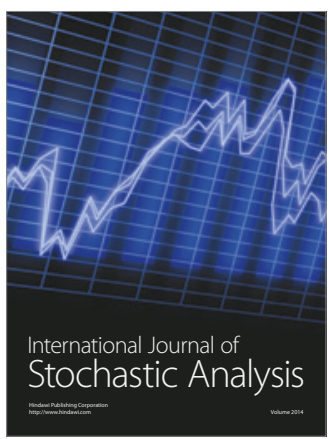

Probability and Statistics
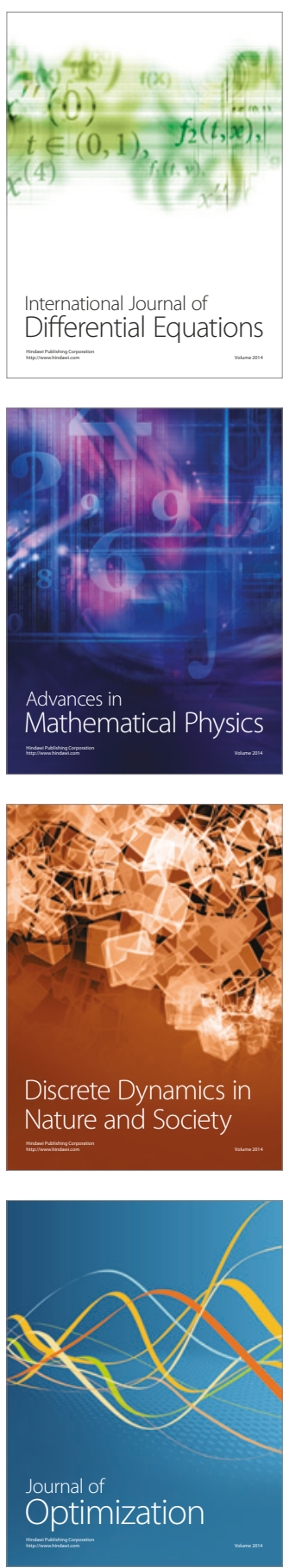